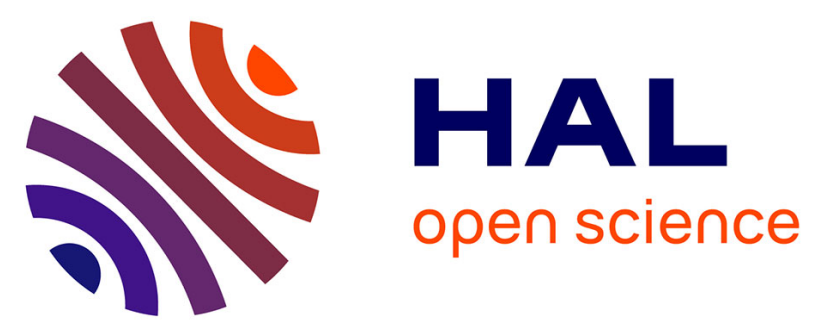

\title{
Measurement of the He-McKellar-Wilkens Topological Phase by Atom Interferometry and Test of Its Independence with Atom Velocity
}

\author{
Jonathan Gillot, S. Lepoutre, A. Gauguet, M. Büchner, J. Vigué
}

\section{To cite this version:}

Jonathan Gillot, S. Lepoutre, A. Gauguet, M. Büchner, J. Vigué. Measurement of the He-McKellarWilkens Topological Phase by Atom Interferometry and Test of Its Independence with Atom Velocity. Physical Review Letters, 2013, 111 (3), pp.030401. 10.1103/physrevlett.111.030401 . hal-03518508

\author{
HAL Id: hal-03518508 \\ https://hal.science/hal-03518508
}

Submitted on 10 Jan 2022

HAL is a multi-disciplinary open access archive for the deposit and dissemination of scientific research documents, whether they are published or not. The documents may come from teaching and research institutions in France or abroad, or from public or private research centers.
L'archive ouverte pluridisciplinaire $\mathbf{H A L}$, est destinée au dépôt et à la diffusion de documents scientifiques de niveau recherche, publiés ou non, émanant des établissements d'enseignement et de recherche français ou étrangers, des laboratoires publics ou privés. 


\title{
Measurement of the He-McKellar-Wilkens Topological Phase by Atom Interferometry and Test of Its Independence with Atom Velocity
}

\author{
J. Gillot, S. Lepoutre, A. Gauguet, M. Büchner, and J. Vigué* \\ Laboratoire Collisions Agrégats Réactivité-IRSAMC, Université de Toulouse-UPS and CNRS, UMR 5589, 31062 Toulouse, France
}

(Received 1 March 2013; published 15 July 2013)

\begin{abstract}
In this Letter, we report a measurement of the He-McKellar-Wilkens (HMW) topological phase by atom interferometry. The experiment is done with our lithium atom interferometer, and in order to suppress the stray effects present in our first experiment, we use optical pumping of the ${ }^{7} \mathrm{Li}$ atoms in their $F=2, m_{F}=+2$ (or -2 ) ground state sublevel. In these conditions, the measured phase shift is the sum of the HMW phase and of the Aharonov-Casher phase, which are separated due to their different $m_{F}$ dependence. The HMW phase has been measured for different lithium beam velocities and the results are in very good agreement with a phase independent of the atom velocity, as expected for a topological phase.
\end{abstract}

DOI: 10.1103/PhysRevLett.111.030401

PACS numbers: 03.65.Vf, 03.75.Dg

In 1959, Aharonov and Bohm discovered the first topological phase [1], a phase shift which appears in the absence of any force acting on the particle, a theoretical discovery confirmed by experiments [2,3]. In 1984, Aharonov and Casher [4] discovered a second topological phase, in the interaction of a magnetic dipole with an electric field, also confirmed by experiments [5-8]. In 1993-1994, by applying Maxwell duality to the Aharonov-Casher phase, $\mathrm{He}$ and McKellar [9] and Wilkens [10] have introduced the topological phase now known as the He-McKellar-Wilkens (HMW) phase; it appears when an electric dipole $\mathbf{d}$ propagates in a magnetic field B:

$$
\varphi_{\mathrm{HMW}}=\oint \mathbf{B} \times \mathbf{d} \cdot d \mathbf{r} / \hbar,
$$

where $\mathbf{r}$ is the position of the particle and the integration path follows the interferometer arms. To observe this phase with an atom interferometer, an electric dipole must be induced in the atom by an electric field [10].

In a previous work [11], we have tested the HMW phase with our lithium atom interferometer [12], using the arrangement of Wei et al. [13]: opposite electric dipoles induced by opposite electric fields on the two interferometer arms interact with a common magnetic field. Our experiment was sensitive enough to detect the HMW phase but important stray phase shifts due to experimental defects were present and we had to develop a model of our experiment to extract the HMW phase. This model was most probably imperfect and this makes the accuracy study difficult, leading to a discrepancy with the theoretical value of $31 \%$. It appeared that the main source of stray phase shifts was the average over the eight hyperfine-Zeeman $F$, $m_{F}$ sublevels of ${ }^{7} \mathrm{Li}$ ground state: each $F, m_{F}$ sublevel gives a contribution to the total fringe signal, with a weight equal to the product of the beam intensity by the fringe visibility and the fringe visibility of such a contribution is sensitive to the correlation of defects. In order to sort out this problem, we had to prepare the atom in a single $F, m_{F}$ sublevel.

We report here a new experiment in which the lithium atomic beam is optically pumped in $F=2, m_{F}=+2$, or $m_{F}=-2$. Another consequence of optical pumping is that the experiment is now sensitive to the Aharonov-Casher (AC) phase [4]:

$$
\varphi_{\mathrm{AC}}\left(F, m_{F}\right)=-\oint \frac{\left[\mathbf{E}(\mathbf{r}) \times \boldsymbol{\mu}\left(F, m_{F}\right)\right] \cdot d \mathbf{r}}{\hbar c^{2}} .
$$

The different $m_{F}$ dependence of the AC and HMW phases will be used to separate these two phases. We briefly recall the principle of the experiment [11], with some details on the modifications. In the interaction region, two plane capacitors sharing a septum as a common electrode [14] produce opposite electric fields on the two interferometer arms and two coils surrounding these capacitors create the

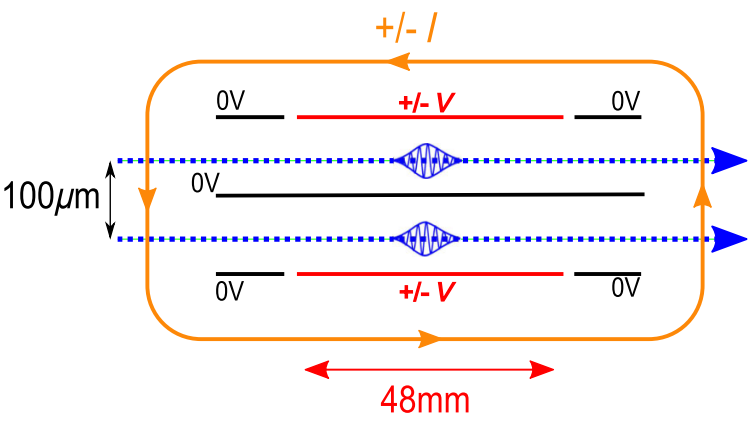

FIG. 1 (color online). Schematic top view of the interaction region (not to scale). The interferometer arms (dotted blue lines) are separated by a septum; the electric fields are produced by two plane capacitors sharing the septum as a common electrode grounded electrode. The high voltage electrodes (in red) are surrounded by grounded guard electrodes (in black). The brown rectangle represents the coils which produce the magnetic field. 
magnetic field needed for the HMW effect (see Fig. 1). The magnetic field of the HMW coils presents a small gradient so that the two interferometer arms see slightly different magnetic fields and this gradient induces a Zeeman phase shift; a compensator coil, placed in another region of the atom interferometer, produces an opposite gradient which is used to cancel the Zeeman phase shift. Finally, the magnitudes of the fields are $E(\mathrm{~V} / \mathrm{m}) \approx 900 \mathrm{~V}$ with the capacitor voltage $V$, up to $800 \mathrm{~V}$ and $B \approx 0.56 I \mathrm{mT}$ with the coil current $I$, up to $40 \mathrm{~A}$. $\varphi_{\mathrm{HMW}}$ is proportional to the integral of the $E B$ product and so to $V I$.

The atomic beam is a supersonic beam with lithium seeded in a noble gas, with a mean velocity $v_{m}$ which scales as $1 / \sqrt{M}$, where $M$ is the atomic mass of the carrier gas. We have varied $v_{m}$ by using krypton $\left(v_{m}=744 \pm\right.$ $18 \mathrm{~m} / \mathrm{s}), \operatorname{argon}\left(v_{m}=1062 \pm 20 \mathrm{~m} / \mathrm{s}\right)$, and neon $\left(v_{m}=\right.$ $1520 \pm 38 \mathrm{~m} / \mathrm{s})$. The beam velocity distribution is close to Gaussian [15] with a $1 / e$ half-width equal to $v_{m} / S_{\|}$where the parallel speed ratio $S_{\|}$varies with the source parameters (nozzle diameter, pressure, temperature, carrier gas), with typical values $S_{\|}=6-8$. The signal intensity is very good with neon and argon but considerably smaller with krypton (see Table I).

Only the most abundant isotope ${ }^{7} \mathrm{Li}$ contributes to the interferometer signal $[12,16]$. The atomic beam is optically pumped in the second vacuum chamber after the skimmer. It is necessary to pump the atoms before collimation to avoid heating of the transverse motion by radiation pressure. We use the $D 1$ line, because the ${ }^{2} P_{1 / 2}$ level has a larger hyperfine structure than the ${ }^{2} P_{3 / 2}$ level. Two circularly polarized laser beams, respectively tuned on the ${ }^{2} S_{1 / 2}$, $F=1 \rightarrow{ }^{2} P_{1 / 2}, F^{\prime}=2$ and $^{2} S_{1 / 2}, F=2 \rightarrow{ }^{2} P_{1 / 2}, F^{\prime}=2$ transitions excite the atoms. The first laser empties the $F=1$ level and the second laser accumulates all the atoms in the $F=2, m_{F}=+2$ sublevel, which is the only sublevel not coupled to the lasers. We have characterized the efficiency of this optical pumping process [17]: the fraction of the population in the $m_{F}=+2$ or -2 sublevel is near $90 \%$ or larger. As discussed above, it is necessary to reverse the $m_{F}$ value to separate the HMW and AC phases. This is done by reversing the magnetic field in the pumping region, so that the $m_{F}$ value changes sign. Then, as the direction of the magnetic field $\mathbf{B}$ is slowly varying in space, an adiabatic argument proves that the projection $m_{F}$ of the

TABLE I. For the three carrier gases, we give the measured value of the mean signal intensity $I_{0}$, the fringe visibility $\mathcal{V}$ for extreme values of the HMW coil current, $I=0$ and $I_{\max }=25 \mathrm{~A}$ for krypton or $I_{\max }=40 \mathrm{~A}$ for argon and neon.

\begin{tabular}{lccc}
\hline \hline Carrier gas & $I_{0}($ atoms $/ \mathrm{s})$ & $\mathcal{V}(I=0)$ & $\mathcal{V}\left(I_{\max }\right)$ \\
\hline Krypton & $0.7 \times 10^{4}$ & $80 \%$ & $26 \%$ \\
Argon & $3.3 \times 10^{4}$ & $75 \%$ & $48 \%$ \\
Neon & $5.6 \times 10^{4}$ & $60 \%$ & $34 \%$ \\
\hline \hline
\end{tabular}

angular momentum $\mathbf{F}$ on an axis parallel to the local field is constant everywhere.

The Zeeman effect of the $F=2, m_{F}=+2$ (or -2 ) sublevel is exactly linear so that the Zeeman phase shift can be exactly compensated by the compensator coil which operates in the field range corresponding to linear Zeeman effect. This better compensation improves the fringe visibility, which makes it possible to apply a larger current $I$, up to $40 \mathrm{~A}$, in the HMW coil. However, there is some residual dispersion of the Zeeman phase shift, because it varies with the altitude of the atom trajectory, and the best we can do is to compensate the mean Zeeman phase shift, its residual dispersion reducing the fringe visibility for large $I$ values. Table I presents typical values of the mean signal intensity $I_{0}$ and of the fringe visibility $V$ measured in low and high magnetic field. The phase sensitivity, which scales like $1 /\left(V \sqrt{I_{0}}\right)$, varies rapidly with the carrier gas and with the HMW coil current.

As in our previous experiment, during each fringe scan, we alternate field configurations in order to eliminate the interferometer phase drifts. We define a field configuration by its $(V, I)$ values, $V$ being the capacitor voltage and $I$ the current in the HMW coil, and we use six configurations: $(0,0),(V, 0),(V, I),(0, I),(-V, 0),(-V, I)$. Fits extract the fringe phase $\varphi(V, I)$ corresponding to each $(V, I)$ configuration. We average about 80 fringe scans, each scan lasting $20 \mathrm{~s}$, to get a phase measurement. Then in successive runs, we record signals for opposite values of the current $I$ and for opposite values of $m_{F}, m_{F}= \pm 2$. As in our previous work, we use the following combination of the measured phases:

$$
\begin{aligned}
\varphi_{E B}\left(V, I, m_{F}\right) & =\varphi_{E+B}(V, I)-\varphi_{E}(V)-\varphi_{B}(I) \\
& =\varphi(V, I)-\varphi(V, 0)-\varphi(0, I)+\varphi(0,0),
\end{aligned}
$$

where $\varphi_{E+B}(V, I)$ is the measured phase shift when the electric and magnetic fields are simultaneously applied, $\varphi_{E}(V)$ is the measured phase shift with the electric field only, and $\varphi_{B}(I)$ is the measured phase shift with the magnetic field only. There are four contributions to the observed phases, namely the Stark phase $\varphi_{S}(V)$, the Zeeman phase $\varphi_{Z}(I)$, the AC phase $\varphi_{\mathrm{AC}}\left(V, I, m_{F}\right)$, and the HMW phase $\varphi_{\text {HMW }}(V, I)$. When $V=0$, the electric field is negligible and the Stark, AC, and HMW phases vanish. When $I=0$, there is still a laboratory field $B_{0}$ and the Zeeman and $\mathrm{AC}$ phases are not vanishing. We get

$$
\begin{aligned}
\varphi_{E B}\left(V, I, m_{F}\right)= & \varphi_{\mathrm{HMW}}(V, I)+\varphi_{\mathrm{AC}}\left(V, I, m_{F}\right) \\
& -\varphi_{\mathrm{AC}}\left(V, I=0, m_{F}\right) .
\end{aligned}
$$

The AC phase, which is proportional to the atom magnetic moment, changes sign with $m_{F}$ and by combining $\varphi_{E B}\left(V, I, m_{F}\right)$ with opposite $m_{F}$ values, we extract the HMW phase:

$$
\varphi_{\mathrm{HMW}}(V, I)=\left[\varphi_{E B}(V, I, 2)+\varphi_{E B}(V, I,-2)\right] / 2 .
$$




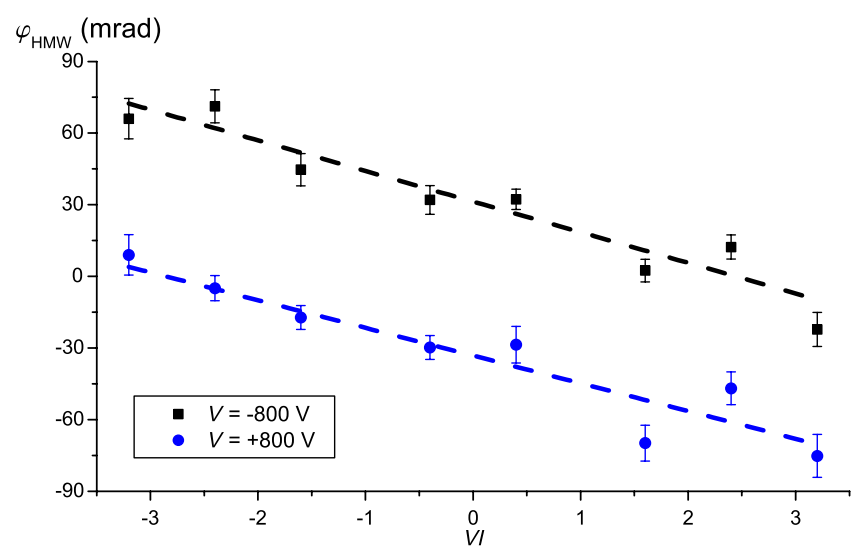

FIG. 2 (color online). The measured phase $\varphi_{\mathrm{HMW}}(V, I)$ given by Eq. (5) is plotted as a function of the $V I$ product, in units of $10^{4} \mathrm{VA}$, for $V=+800 \mathrm{~V}$ and $V=-800 \mathrm{~V}$.

There is a small systematic error in this extraction of $\varphi_{\mathrm{HMW}}(V, I)$ because the optical pumping efficiencies in $m_{F}=+2$ and $m_{F}=-2$ are slightly different. This residual AC phase is always smaller than $10 \mathrm{mrad}$ and depends only on the applied voltage $V$. Thus it appears as an offset and it cannot modify the slope of the HMW phase $\varphi_{\mathrm{HMW}}(V, I)$ with the current $I$. In Fig. 2, we plot the measured values $\varphi_{\mathrm{HMW}}(V, I)$ as a function of the $V I$ product for $V=$ $\pm 800 \mathrm{~V}$ : the variations are well represented by an equation of the form $\varphi_{\mathrm{HMW}}(V, I)=\alpha(V I)+\beta$ with an offset $\beta$. This offset $\beta$ is due to a phase shift which has already been detected in our previous paper (see Fig. 7 of the Supplemental Material belonging to Ref. [11]). Although we have no explanations for this phase shift, extra measurements proved that this phase is not topological and does not depend on the current $I$ in a wide range. In addition, it is an odd function of the applied voltage $V$. As a consequence, it cannot be the residual AC phase discussed above. We also stress that its presence does not affect the HMW phase, which appears in the slope $\alpha$ of the straight line.

For all the data, we used similar fit procedures to those presented in Fig. 2 and, for each series of data, we correct the measured $\varphi_{\mathrm{HMW}}(V, I)$ values by subtracting the offset $\beta$ of this series to get $\varphi_{\mathrm{HMW}}^{c}(V, I)=\varphi_{\mathrm{HMW}}(V, I)-\beta$. In Fig. 3, we plot all the corrected values $\varphi_{\mathrm{HMW}}^{c}(V, I)$ as a function of the $V I$ product. The slope of $\varphi_{\mathrm{HMW}}^{c}(V, I)$ vs $V I$ is worked out from a linear fit of all the data points:

$$
\begin{aligned}
& \varphi_{\mathrm{HMW}}^{c}(V, I) /(V I)=(1.41 \pm 0.24) \times 10^{-6} \mathrm{rad} / \mathrm{VA} \\
& \quad \text { for } v_{m}=744 \pm 18 \mathrm{~m} / \mathrm{s}, \\
& \varphi_{\mathrm{HMW}}^{c}(V, I) /(V I)=(1.315 \pm 0.071) \times 10^{-6} \mathrm{rad} / \mathrm{VA} \\
& \quad \text { for } v_{m}=1062 \pm 20 \mathrm{~m} / \mathrm{s}, \\
& \varphi_{\mathrm{HMW}}^{c}(V, I) /(V I)=(1.270 \pm 0.072) \times 10^{-6} \mathrm{rad} / \mathrm{VA} \\
& \quad \text { for } v_{m}=1520 \pm 38 \mathrm{~m} / \mathrm{s},
\end{aligned}
$$

where the error bars are statistical $1 \sigma$ error bars.
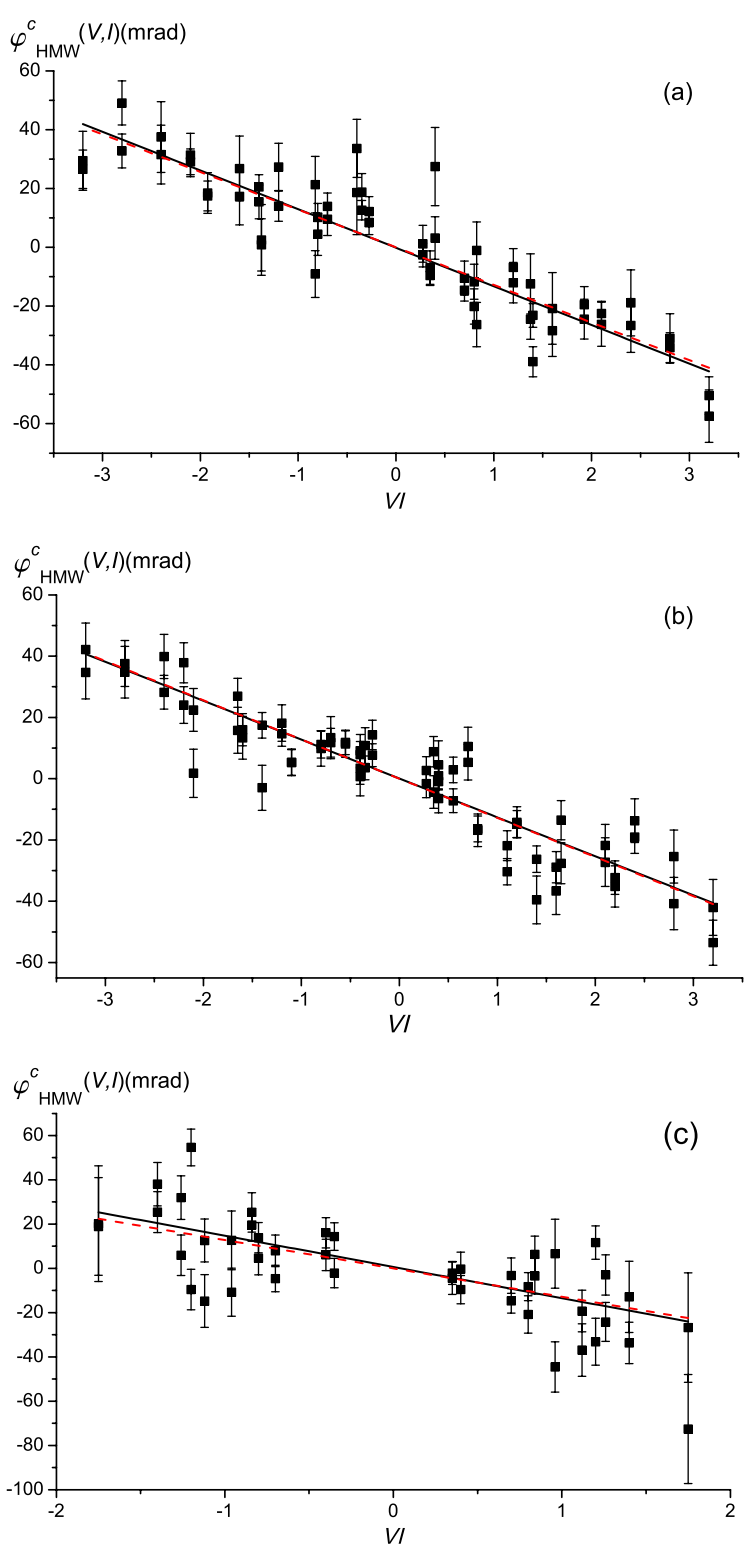

FIG. 3 (color online). The corrected HMW phase $\varphi_{\mathrm{HMW}}^{c}(V, I)$ is plotted as a function of the $V I$ product (in units of $10^{4} \mathrm{VA}$ ). Panel (a) experiment with argon as carrier gas, $v_{m}=1062 \pm$ $20 \mathrm{~m} / \mathrm{s}$; panel (b) experiment with neon as carrier gas, $v_{m}=$ $1520 \pm 38 \mathrm{~m} / \mathrm{s}$; panel (c) experiment with krypton as carrier gas, $v_{m}=744 \pm 18 \mathrm{~m} / \mathrm{s}$. The full (black) line is the best fit while the predicted value of $\varphi_{\mathrm{HMW}}$ is represented by a dashed (red) line: these two lines almost coincide except in panel (c).

Using the theoretical expression, Eq. (1), lithium atom polarizability $\alpha_{\mathrm{Li}}[18,19]$ and the geometry of the capacitors and of the HMW coil [11], we can predict the value of HMW phase $\varphi_{\mathrm{HMW}}(V, I)(\mathrm{rad})=(1.28 \pm 0.03) \times 10^{-6} V I$ with $V I$ in VA. The uncertainty, near $2.5 \%$, comes solely from the geometry of the capacitors and of the HMW coil, the theoretical $\alpha_{\mathrm{Li}}$ value being very accurate. The three measured slopes are in very good agreement with the expected value. Moreover, these slopes are independent 


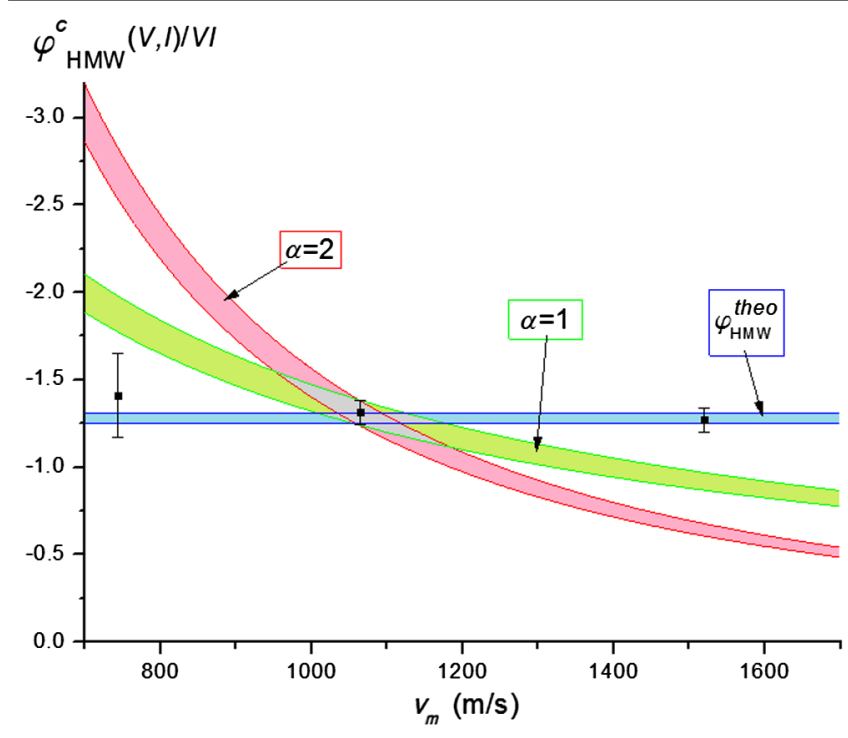

FIG. 4 (color online). Plot of $\varphi_{\mathrm{HMW}}^{c}(V, I) /(V I)$ (in units of $\left.10^{-6} \mathrm{rad} / \mathrm{VA}\right)$ as a function of the mean atomic beam velocity $v_{m}$ : the experimental results are compared to the theoretical value, represented with its error bar by the (blue) horizontal band. The shaded areas represent what would be the phase if, starting from its value at $1062 \mathrm{~m} / \mathrm{s}$, the HMW phase was varying like $1 / v_{m}^{\alpha}$ with $\alpha=1$ (green) or $\alpha=2$ (pink).

of the atom velocity, in agreement with the fundamental property of a topological phase. In Fig. 4, a potential velocity scaling in $1 / v_{m}$ or $1 / v_{m}^{2}$, which are the velocity dependence of dynamical phases, either due to an homogeneous perturbation applied to one interferometer arm $\left(1 / v_{m}\right)$ or to a uniform force field applied to both arms $\left(1 / v_{m}^{2}\right)$ [20]. Clearly, these three data points completely rule out a $1 / v_{m}$ dependence and, more strongly, a $1 / v_{m}^{2}$ dependence.

In conclusion, we measured the He-McKellar-Wilkens phase by atom interferometry. We have improved our first experiment by an optical pumping of the lithium atomic beam in the $F=2, m_{F}=+2$ (or -2 ) ground state sublevel. This pumping provides two main advantages: the stray phase shifts due to averaging over the $F, m_{F}$ sublevels have disappeared and it has been possible to operate with larger magnetic fields because the fringe visibility remains good up to large fields. The Aharonov-Casher phase, which now contributes to the measured phase shift, is separated from the HMW phase by combining experiments with opposite $m_{F}$ levels.

We have observed in the present experiment a phase shift odd in the applied voltage and independent of the magnetic field. This phase shift, which was already observed in our previous experiment, appears as an offset of the measurement of the HMW phase and it is easy to subtract this offset. The resulting data set is of good quality and we have measured the slope $\varphi_{\mathrm{HMW}}^{c}(V, I) /(V I)$ for three mean velocities $v_{m}$ of the lithium beam, covering the range
744-1520 m/s. These three measurements agree very well with the expected theoretical value of the HMW phase, calculated from the geometry of our experiment, and they are also fully consistent with the independence of a topological phase with atom velocity.

We thank the laboratory technical staff for their help, A. Cronin and B.H. J. McKellar for fruitful discussions, G. Trénec, A. Miffre, and M. Jacquey for all the work done on our atom interferometer. We thank CNRS INP, ANR (Grants No. ANR-05-BLAN-0094 and No. ANR-11BS04-016-01 HIPATI) and Région Midi-Pyrénées for support.

*jacques.vigue@irsamc.ups-tlse.fr

[1] Y. Aharonov and A. Bohm, Phys. Rev. 115, 485 (1959).

[2] R. G. Chambers, Phys. Rev. Lett. 5, 3 (1960).

[3] A. Tonomura, N. Osakabe, T. Matsuda, T. Kawasaki, J. Endo, S. Yano, and H. Yamada, Phys. Rev. Lett. 56, 792 (1986).

[4] Y. Aharonov and A. Casher, Phys. Rev. Lett. 53, 319 (1984).

[5] A. Cimmino, G. I. Opat, A. G. Klein, H. Kaiser, S. A. Werner, M. Arif, and R. Clothier, Phys. Rev. Lett. 63, 380 (1989).

[6] K. Sangster, E. A. Hinds, S. M. Barnett, and E. Riis, Phys. Rev. Lett. 71, 3641 (1993); K. Sangster, E. A. Hinds, S. M. Barnett, E. Riis, and A. G. Sinclair, Phys. Rev. A 51, 1776 (1995).

[7] A. Görlitz, B. Schuh, and A. Weis, Phys. Rev. A 51, R4305 (1995).

[8] K. Zeiske, G. Zinner, F. Riehle, and J. Helmcke, Appl. Phys. B 60, 205 (1995).

[9] X.-G. He and B.H. J. McKellar, Phys. Rev. A 47, 3424 (1993).

[10] M. Wilkens, Phys. Rev. Lett. 72, 5 (1994).

[11] S. Lepoutre, A. Gauguet, G. Trénec, M. Büchner, and J. Vigué, Phys. Rev. Lett. 109, 120404 (2012).

[12] A. Miffre, M. Jacquey, M. Büchner, G. Trénec, and J. Vigué, Eur. Phys. J. D 33, 99 (2005).

[13] H. Wei, R. Han, and X. Wei, Phys. Rev. Lett. 75, 2071 (1995).

[14] C. R. Ekstrom, J. Schmiedmayer, M. S. Chapman, T.D. Hammond, and D. E. Pritchard, Phys. Rev. A 51, 3883 (1995).

[15] H. Haberland, U. Buck, and M. Tolle, Rev. Sci. Instrum. 56, 1712 (1985).

[16] M. Jacquey, A. Miffre, M. Büchner, G. Trénec, and J. Vigué, Europhys. Lett. 77, 20007 (2007).

[17] J. Gillot, A. Gauguet, M. Büchner, and J. Vigué, Eur. Phys. J. D (to be published).

[18] A. Miffre, M. Jacquey, M. Büchner, G. Trénec, and J. Vigué, Eur. Phys. J. D 38, 353 (2006).

[19] M. Puchalski, D. Kedziera, and K. Pachucki, Phys. Rev. A 84, 052518 (2011); 85, 019910(E) (2012).

[20] A. D. Cronin, J. Schmiedmayer, and D. E. Pritchard, Rev. Mod. Phys. 81, 1051 (2009). 